\title{
Expression and clinical significance of c-Met in advanced esophageal squamous cell carcinoma
}

\author{
Yingying $\mathrm{Xu}^{1 \dagger}$, Zhi Peng ${ }^{1 \dagger}$, Zhongwu $\mathrm{Li}^{2}$, Ming Lu'${ }^{1}$, Jing Gao ${ }^{1}$, Yilin $\mathrm{Li}^{1}$, Yanyan $\mathrm{Li}^{1}$ and Lin Shen ${ }^{1 *}$
}

\begin{abstract}
Background: c-Met, one of current potential hot targets, has been suggested as a potential tumor marker in the development of esophageal squamous cell carcinoma (ESCC). Our aim was to investigate the expression of c-Met in advanced esophageal squamous cell carcinoma in four phase II trials who had tumor tissues from archival in our center and analyze the correlations between c-Met expression and clinical features.

Methods: Ninety patients with advanced ESCC who were admitted to the phase II clinical trials in the Department of Gastrointestinal Oncology, Peking University Cancer Hospital and Institute from March 2007 to March 2014 were finally eligible for present study and the corresponding tissues and clinical data were collected. The expression of c-Met in the tissue samples was detected by immunohistochemistry (IHC). c-Met overexpression was defined as $\geq$ the median value of $\mathrm{H}$-score. Kaplan-Meier and Cox multivariate regression were conducted to evaluate the relationship between c-Met expression and ESCC survival.
\end{abstract}

Results: The overexpression of c-Met is $43.3 \%$ in advanced ESCC. There was no statistical difference between c-Met expression and clinical features except sex and tumor location. Survival analysis documented that the overexpression of c-Met predicted a worse prognosis (OS: $253 \mathrm{~d}$ vs $422 \mathrm{~d}, P=0.011$ ). In the group treated with chemotherapy combined with anti-EGFR drugs, patients with lowexpression of c-Met had a better OS than those with overexpression of c-Met (OS: 577 d vs $232 \mathrm{~d}, P=0.007$ ).

Conclusions: c-Met may be an independent prognostic factor in advanced ESCC. The overexpression of c-Met may predict a worse efficacy of anti-EGFR therapy.

Keywords: Esophageal squamous cell carcinoma, c-Met, Chemotherapy, Overall survival

\section{Background}

Esophageal carcinoma is one of the most common gastrointestinal cancers, the incidence of which ranks the fifth among all of the malignant cancers in China [1]. More than $95 \%$ of esophageal cancer in China is esophageal squamous cell carcinoma (ESCC). Due to the atypical clinical symptoms, most patients develop into advanced stages when first diagnosed and lost operation opportunity. Systematic therapies, including chemotherapy, are of great importance for patients with advanced ESCC, whereas the efficacy of chemotherapy based on 5FU/DDP/PTX is limited. As the ubiquity poor prognosis

\footnotetext{
*Correspondence: lin100@medmail.com.cn

${ }^{\dagger}$ Equal contributors

'Department of Gastrointestinal Oncology, Key Laboratory of Carcinogenesis and Translational Research (Ministry of Education), Peking University Cancer Hospital \& Institute, FuCheng Road 52, HaiDian District, Beijing, China Full list of author information is available at the end of the article
}

of advanced ESCC, new strategies are urgently needed, especially of targeted-therapy. c-Met is one of the most important factors with cancer-associated receptors and pathways. c-Met tyrosine kinase is the cell-surface receptor for hepatocyte growth factor (HGF) which is involved in regulating cell proliferation, apoptosis, and migration. c-Met activity is normally detected in defined stages of embryogenesis and organogenesis [2-7]. HGF and c-Met have a significant relevance to lymph node stage and distant metastasis. It was reported that c-Met was involved in a number of human primary tumors, including gastric, breast, colorectal, liver and renal cancer [8].

Recently, a meta-analysis focusing on c-Met overexpression and the prognosis of gastric cancer including 14 studies [9] indicated that higher amplification and expression of c-Met gene in gastric cancer is an indicator of poor prognosis. c-Met was overexpressed in 34\%-54\%

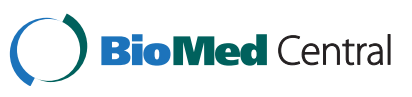


of esophageal adenocarcinoma and had a significant association with survive [10]. c-Met overexpression in ESCC is about $7 \%$ and not correlated with prognosis in western countries. Our study in China showed that c-Met overexpression in ESCC is about 34\% [11], which differs from western countries. However, by now, there is no study on the c-Met expression and clinical significance in advanced ESCC.

Therefore, in this study, we detected c-Met expression in advanced ESCC patients who involved in the phase II clinical trials in our center. We investigated the expression levels of c-Met in advanced ESCC and estimated the relationship between c-Met expression and clinical features.

\section{Methods}

\section{Patients and tumor specimens}

Tumor samples were retrospectively identified from patients with ESCC who were enrolled in four phase II clinical trials between 2007 and 2014 at the Peking University Cancer Hospital in Beijing, China. Four phase II clinical trials for first-line treatment of ESCC were involved: Paclitaxel and Cisplatin in Patients With Advanced Squamous-Cell Carcinoma of the Esophagus [12]; Irinotecan Combined With Cisplatin as 1st Line Treatment for Esophageal Squamous Cell Cancer: a Single Center Prospective Clinical Trial (NCT01051765); Study of Nimotuzumab to Treat Esophageal Squamous Cell Carcinoma (NCT01993784); Sequential Paclitaxel Chemotherapy and Radiotherapy as 1st Line Treatment for Elderly Esophageal Squamous Cell Cancer (NCT02016287). The inclusion and exclusion criteria were as follows:

Inclusion criterion:

1. Histological confirmed stage IV according to AJCC 6.0 or recurrent ESCC.

2. Patients have not received any palliative chemotherapy or radiotherapy before.

3. Adjuvant therapies should be received more than 6 months before recurrence.

\section{Exclusion criterion:}

1. Adjuvant therapies are received within 6 months before recurrence.

2. Patients who have other primary carcinomas except ESCC.

3. Histology confirmed mixed tumor.

Anti-total c-Met (SP44) rabbit monoclonal primary antibodies were purchased from Ventana Company (Ventana Medical System, Tucson, USA).

Tissue samples for diagnostic purposes were obtained with the consent of each patient. All tumor specimens were fixed in $10 \%$ buffered formalin, embedded in paraffin and then made into continuous $4 \mu \mathrm{m}$ tissue sections for IHC examination.

All patients signed written informed consent for their information to be used for study. Study approval was obtained from independent ethics committees at Peking University Cancer Hospital. The study was undertaken in accordance with the ethical standards of the World Medical Association Declaration of Helsinki.

\section{Immunohistochemistry (IHC)}

IHC evaluation was performed using anti-total c-Met (SP44) rabbit monoclonal primary antibody. The staining was carried out according to the manufactures' protocol on the BenchMark XT platform from Ventana utilizing the ultraView detection kit. The c-Met staining intensities were evaluated by two pathologists who were blinded to the diagnosis of individual patients. c-Met was localized primarily in the cytoplasm and membrane. Specifically, intensity was scored according to a four-tier systems: 0 , no staining; $1+$, weak; $2+$, moderate; and $3+$, strong.

To our knowledge, there were no validated scoring systems for interpretation of c-Met staining intensity. Here, positive results were judged by two systems of criterion.

1. $\mathrm{H}$-score assessment was based on staining intensity (0-3) and the percentage of positive cells (0-100\%). Each individual intensity level was multiplied by the percentage of cells and all values were added to obtain the final IHC score, ranging from 0 to 300 . The final score was calculated from the scores of assessment at membranous and cytoplasmic expression.

2. The other evaluation of c-Met was performed according to MetMab IHC defined scoring criteria (positively defined as having $\geq 50 \%$ of tumor cells positive for membranous or cytoplasmic and/or c-Met immunostaining with moderate or strong intensity, i.e. $\geq 2+$ ) in the clinical trial of NCT01456325, a MetMab phase III trial in advanced NSCLC [13].

\section{Statistical analysis}

A number of prognostic factors between different c-Met expression groups were examined in univariate analysis. These factors included: (1) sex: male and female; (2) baseline age, dichotomized as $\leq 59$ years vs. $>59$ years; (3) tumor location; (4) presence of distant metastasis; and (5) presence of lymph node invasion. Comparison of categorical variables was performed with the chi-square test or the Fisher's exact test. Initial analysis involved a univariate investigation of relationship between c-Met overexpression and OS time using Kaplan-Meier survival curves. To identify independent biomarkers, multivariate analyses adjusted for the baseline variables were performed using a Cox regression model for OS. $P$ values 


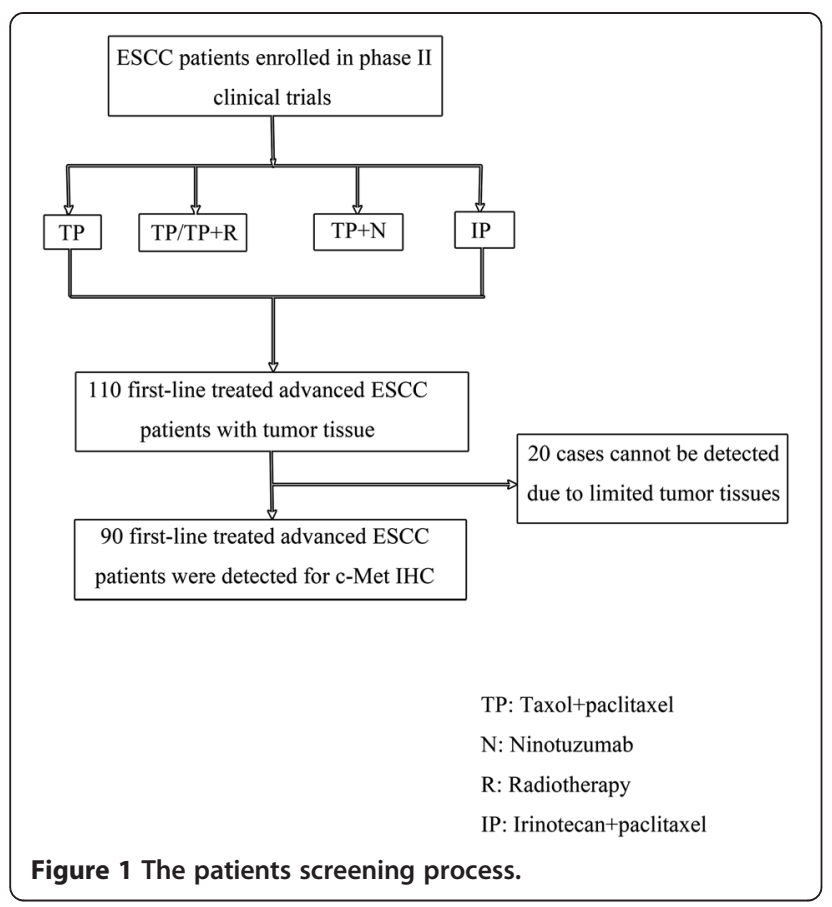

of $<0.05$ were considered significant. All analysis were performed using SPSS 19.0 software (IBM, Armonk, NY).

\section{Results}

\section{Patients' characteristics and c-Met expression}

After screening all the patients' medical record, one hundred and ten cases were detected for c-Met IHC (Figure 1). Due to limited tumor tissues, ninety cases were successfully detected. The representative IHC intensity of c-Met expression is described in Figure 2. All patients were followed up till Nov 6, 2014. Of the 90 patients, 63 died, 19 survived, and 8 were lost.

$\mathrm{H}$-score of c-Met IHC ranged from 0 to 270 , with the median value of 20 , which was chosen as the cutoff point for separating c-Met over-expression tumors from c-Met low-expression tumor. Of the 90 patients, 51 cases had an $\mathrm{H}$-score $\leq 20$, considered as IHC low-expression, and 39 cases had an $\mathrm{H}$-score $>20$, considered as IHC over-expression. No statistically significant difference of c-Met expression was found between different groups of sex, age, tumor location, tumor differentiation, lymph node invasion and distant metastasis (Table 1).

\section{c-Met expression correlated with patient survival in advanced ESCC}

The relationship between OS and clinical features of ESCC patients was analyzed through log-rank and chisquare test. The results showed that no statistical significance of OS was found in different groups of age, stage, distant metastasis, tumor differentiation. However, OS was significantly different by sex and tumor location. Female patients had a better prognosis than male patients (577d vs 333d, $P=0.041)$. Patients with tumor located in upper thoracic (475d) and middle thoracic (422d) had a better prognosis than those in lower thoracic $(258 \mathrm{~d})(P=0.005)$.

Progress-free survival(PFS) was not significantly different between the two c-Met expression groups (the median PFS of c-Met over-expression vs c-Met low-expression:

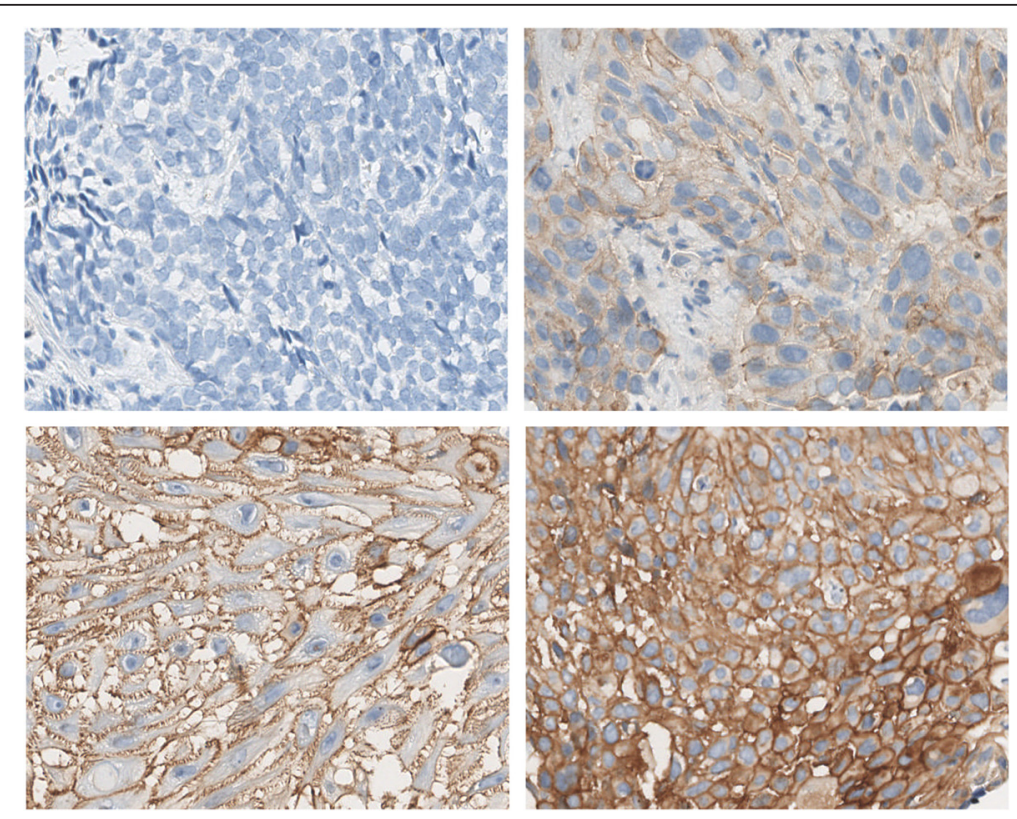

Figure 2 The representative c-Met staining intensities were localized primarily in the cytoplasm and membrane. Intensity: 0 , no staining; 1+, weak; 2+, moderate; and 3+, strong. 
Table 1 The relationship between c-Met expression and clinical features of ESCC (according to H-score)

\begin{tabular}{|c|c|c|c|c|}
\hline & $\mathbf{N}$ & H-score $\leq 20$ & H-score > 20 & $P$ \\
\hline \multicolumn{5}{|l|}{ Sex } \\
\hline Male & 73 & 39 & 34 & \multirow[t]{2}{*}{0.198} \\
\hline Female & 17 & 12 & 5 & \\
\hline \multicolumn{5}{|l|}{ Age } \\
\hline$\leq 59$ & 45 & 25 & 20 & \multirow[t]{2}{*}{0.832} \\
\hline$>59$ & 45 & 26 & 19 & \\
\hline \multicolumn{5}{|l|}{ Tumor location } \\
\hline Upper thoracic & 6 & 4 & 2 & \multirow[t]{4}{*}{0.489} \\
\hline Middle thoracic & 48 & 24 & 24 & \\
\hline Lower thoracic & 35 & 22 & 13 & \\
\hline Abdominal & 1 & 1 & 0 & \\
\hline \multicolumn{5}{|c|}{ Tumor differentiation } \\
\hline Poor & 31 & 16 & 15 & \multirow[t]{3}{*}{0.769} \\
\hline Moderate & 46 & 27 & 19 & \\
\hline Well & 13 & 8 & 5 & \\
\hline \multicolumn{5}{|l|}{ Distant metastasis } \\
\hline Yes & 27 & 13 & 14 & 0.286 \\
\hline Liver & 14 & 8 & 6 & 0.969 \\
\hline Lung & 19 & 8 & 11 & \multirow[t]{2}{*}{0.114} \\
\hline No & 64 & 38 & 25 & \\
\hline \multicolumn{5}{|c|}{ Lymph node invasion } \\
\hline Yes & 85 & 48 & 37 & \multirow[t]{2}{*}{0.877} \\
\hline No & 5 & 3 & 2 & \\
\hline
\end{tabular}

$188 \mathrm{~d}$ vs $178 \mathrm{~d}, P=0.089)$. The median OS was $333 \mathrm{~d}$ (95\% CI 252d-482d). The OS was closely related to the expression of c-Met in ESCC tissues. As shown in Figure 3, the survival rate of c-Met over-expression patients was significantly lower than that in the low-expression group (253d vs $422 \mathrm{~d}, P=0.011)$. In the multivariate Cox regression model adjusted for baseline variants, there was still a statistical significance between OS and c-Met expression $(\mathrm{HR}=1.805,95 \% \mathrm{CI}=1.045-3.117, P=0.034)$.

In this study, 25 patients received chemotherapy of taxol and cisplatin (TP) plus nimotuzumab, 55 patients received chemotherapy of $\mathrm{TP}$ alone, and 10 patients received other chemotherapies. In c-Met overexpression group, OS of patients who received TP plus nimotuzumab was not different from that of patients who received TP alone (232d vs 258d, $P=0.221)$. Similar results were obtained in c-Met low-expression group $(577 \mathrm{~d} v s$ 422d, $P=0.152$ ). In TP plus nimotuzumab group, the OS of patients with c-Met low-expression was significantly better than those with c-Met over-expression (577d vs 232d, $P=0.007)$. In TP alone group, no statistical significance was found (422d $v s 258 \mathrm{~d}, P=0.076$ ) (Figure 4).

The other evaluation of c-Met was performed according to MetMab IHC defined scoring criterion. According to this criterion, 16 patients $(17.8 \%)$ had c-Met overexpression and 74 patients (82.2\%) had c-Met lowexpression. There was no significant relevance between c-Met expression and different groups of age, sex, tumor location, tumor differentiation, distant metastasis. The patients with c-Met low-expression had a trend of better prognosis with no statistical significance $(P=0.289)$.
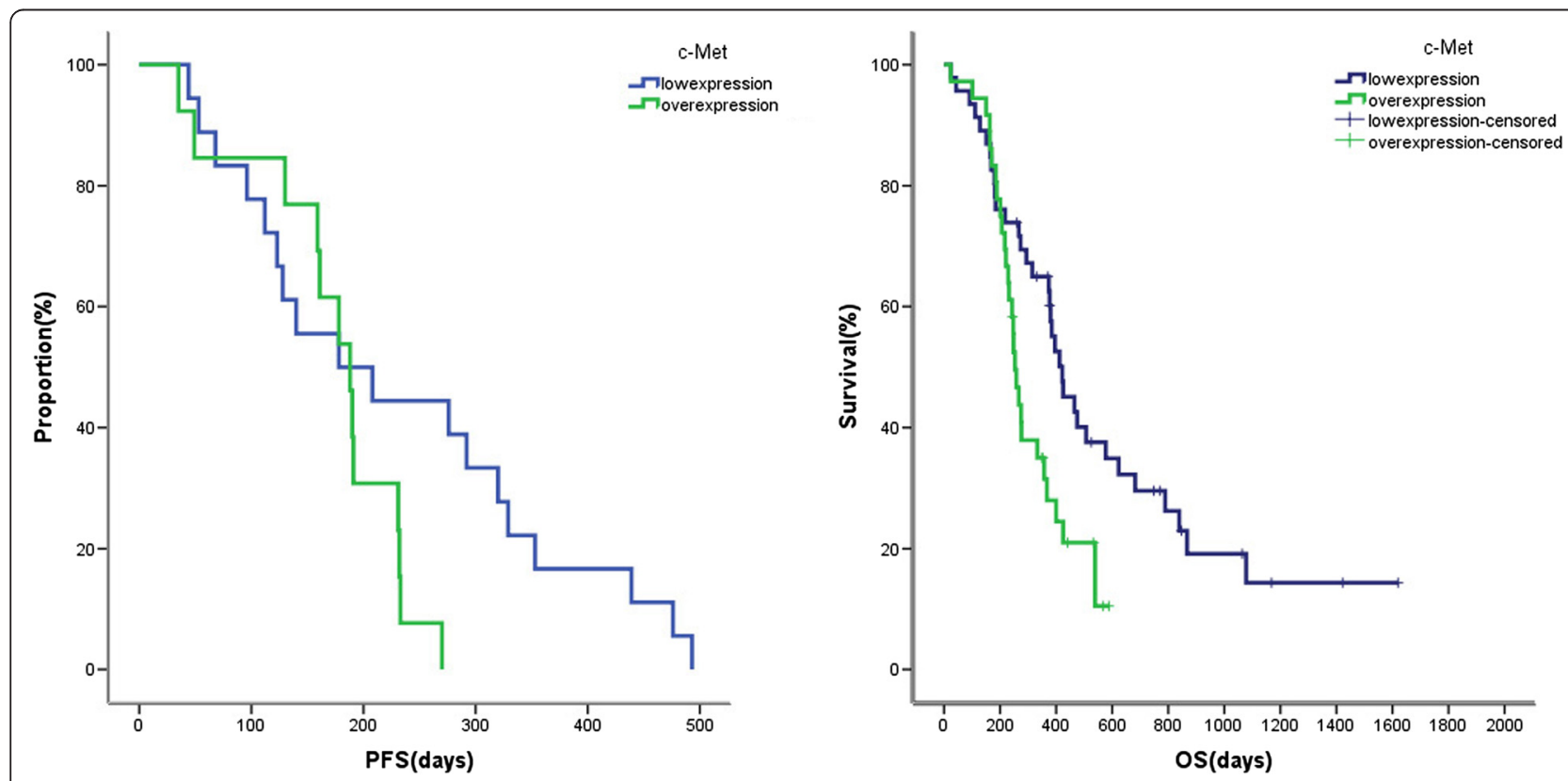

Figure 3 Kaplan-Meier survival curves of patients with ESCC according to c-Met expression. 


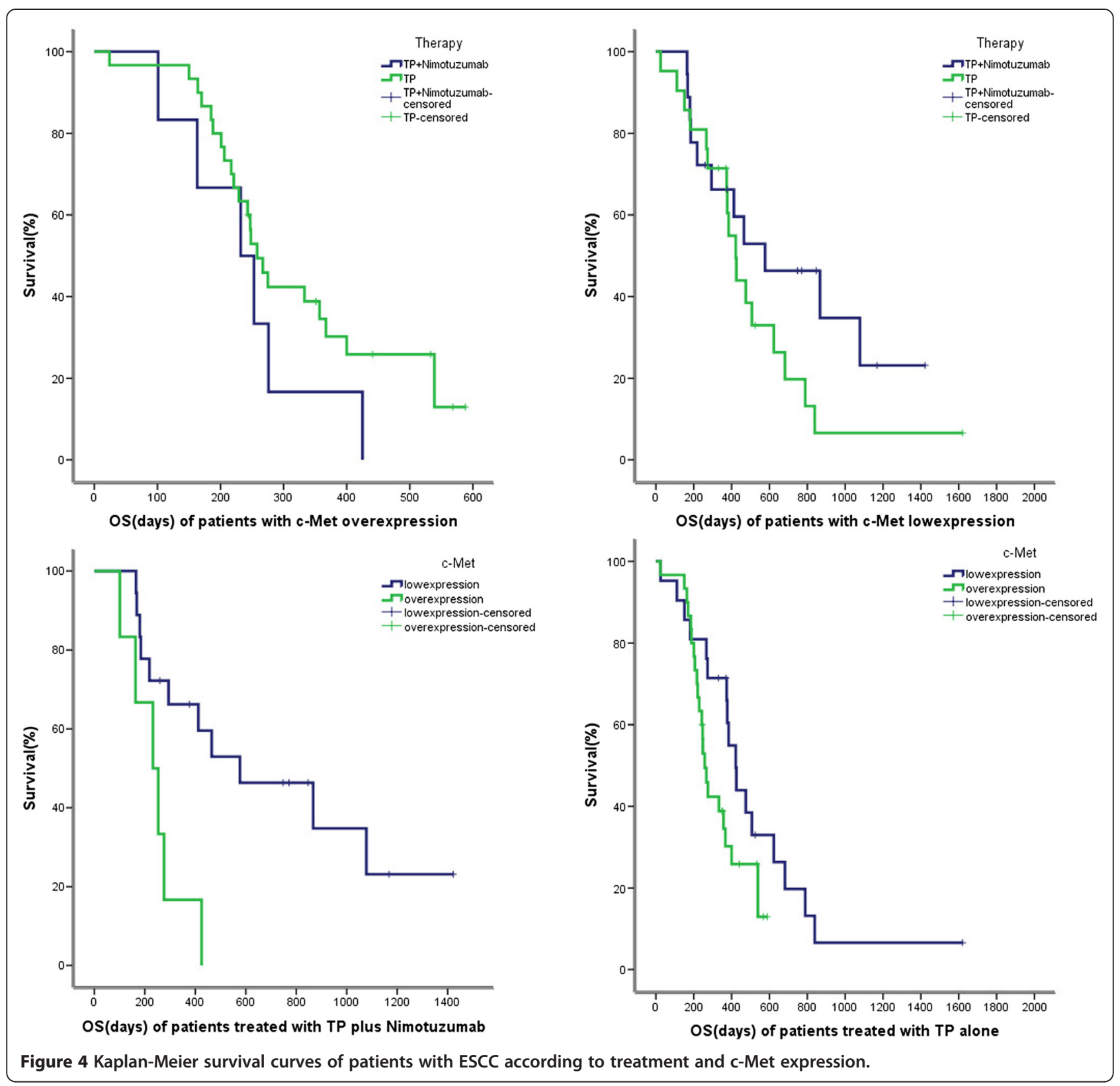

\section{Discussion}

c-Met expression has been reported in a number of human primary tumors, including gastric, breast, colorectal, liver and renal cancer. c-Met plays an important role in tumor development and metastasis [8]. As the only receptor of HGF, c-Met kinase activation resulting in activation of downstream signaling which intermediates such as mitogen-activated protein kinase (MAPK), mammalian target of rapamycin (mTOR) pathway, and signal transducer and activator of transcription (STAT) pathway which leads to changes in gene expression and cell behavior, like increased proliferation, survival, motility, invasiveness, and stimulation of angiogenesis.
Our study detected c-Met expression in 90 ESCC patients and analyzed the relationship between c-Met expression and clinical features and prognosis. According to H-score assessment, $43.3 \%$ of patients had c-Met overexpression, similar to other reports in China [11]. The most important finding in this study was that c-Met overexpression was associated with shorter OS in the patients with advanced ESCC, which may give clues for target therapy in advanced ESCC.

One study reported that an increased expression of cMet was seen along the metaplasia-adenocarcinoma sequence and patients with esophageal adenocarcinoma with c-Met positive tumors showed lower 6-month 
survival rates after surgical resection than those with cMet negative tumors [14]. There were few reports on ESCC in western countries. Mesteri reported that 7.6\% of ESCC patients had c-Met over-expression, but c-Met plays no relevant role in ESCC [10]. However, there are no metastatic patients included in this study and the difference between stages may cause the different results. In China, more than 95\% esophageal cancer patients are ESCC. c-Met overexpression of ESCC in China ranges from $34 \%$ to $58 \%$. Our study had similar results. Dong G finds [15] that metastatic SCC cells that overexpress c-Met exhibit angiogenesis factor expression and enhance scattering in response to HGF in vitro, and tumorigenesis and metastasis in response to HGF in the tumor microenvironment in vivo. Peng [9] did a meta-analysis on c-Met expression in gastric carcinoma and found that higher c-Met gene amplification and expression in gastric cancer was an indicator of poor prognosis. Up to now, there is no other study focused on the relationship between c-Met expression and prognosis in ESCC in China. Further research still needs.

In our study, patients with c-Met lowexpression had a better OS than those with c-Met overexpression both in TP plus nimotuzumab group and in TP group. In c-Met overexpression group, patients treated with TP plus nimotuzumab had a worse OS than those treated with $\mathrm{TP}$ alone. On the contrary, in c-Met lowexpression group, patients treated with TP plus nimotuzumab had a better OS than those treated with TP alone. This suggests that patients with c-Met overexpression may not be suitable for anti-EGFR treatment. There has been no study on anti-EGFR treatment and c-Met expression before. However, a study finds out that in lung cancer, amplification of c-Met causes gefitinib resistance by driving ERBB3-dependent activation of PI3K, a pathway thought to be specific to EGFR/ERBB family receptors [16]. Recently, another study in colorectal cancer highlights the role of c-Met in mediating primary and secondary resistance to anti-EGFR therapies and encourages the use of c-Met inhibitors in patients displaying resistance as a result of c-Met amplification [17]. In the breast cancer, c-Met contributes to trastuzumab resistance, as inhibition of c-Met sensitizes cells to trastuzumab-mediated growth inhibition [18]. High gene copy numbers of c-Met and HGF associate with an increased risk of trastuzumab-based therapy failure in HER2-positive metastatic breast cancer [19]. In gastric cancer, HGF activation of c-Met receptors rescues cells from lapatinib-induced growth inhibition by restimulating the downstream pathways and restoring normal cellcycle progression. This rescue effect could be abrogated by inhibiting c-Met with PHA-665752 (a highly specific c-Met inhibitor) or down-regulating c-Met expression with siRNA [20]. These mechanisms above may exist in
ESCC which cause anti-EGFR drugs resistance. Further confirmation is needed.

There were some limitations in this study. First, this study was conducted in a relatively small sample size. Secondly, due to a retrospective study, only cancer tissues before treatment were detected. It would be better that cancer tissues after treatment, lymph nodes, distant metastasis, and normal tissues adjacent to cancer could be detected for c-Met IHC and c-Met gene amplification. On the other hand, information of patients treated with different therapies was not well matched. Thus, our findings should be validated in subsequent prospective studies in future before clinical application.

In short, c-Met plays an important role in development of ESCC and may have an effect on anti-EGFR therapy, which needs further studies.

\section{Conclusion}

Patients with c-Met over-expression had a poorer prognosis than those with c-Met low-expression, suggesting that c-Met may be an independent prognostic biomarker in ESCC. Patients with c-Met over-expression treated with nimotuzumab had a shorter OS, indicating c-Met might be a predictor of efficacy of anti-EGFR therapy.

\section{Competing interests}

The authors declare that they have no competing interests.

\section{Authors' contributions}

$X-Y Y, P Z$, and $S L$ designed research; $X-Y Y, P Z, L-Y L$, and $L-Y Y$ conducted research; $X-Y Y, P Z, M L$, and $L-Z W$ analyzed data; $X-Y Y, P Z$, and $G$ J wrote the draft; all authors read, reviewed and approved the final manuscript. SL had primary responsibility for final content.

\section{Acknowledgements}

This work was supported by National Natural Science Foundation of China (No. 81172110), National High Technology Research and Development Program (No. 2012AA02A504), Beijing Municipal Science \& Technology Commission Program (No. Z11110706730000), Beijing Natural Science Foundation (7142034), and China Postdoctoral Science Foundation (2013 M530494).

\section{Author details}

'Department of Gastrointestinal Oncology, Key Laboratory of Carcinogenesis and Translational Research (Ministry of Education), Peking University Cancer Hospital \& Institute, FuCheng Road 52, HaiDian District, Beijing, China. 2Department of Pathology, Key Laboratory of Carcinogenesis and Translational Research (Ministry of Education), Peking University Cancer Hospital \& Institute, Beijing, China.

Received: 3 August 2014 Accepted: 22 December 2014

Published online: 15 January 2015

\section{References}

1. Jemal A, Bray F, Center MM, Ferlay J, Ward E, Forman D. Global cancer statistics. CA:Cancer J Clin. 2011;61(2):69-90.

2. Bladt F, Riethmacher D, Isenmann S, Aguzzi A, Birchmeier C. Essential role for the c-met receptor in the migration of myogenic precursor cells into the limb bud. Nature. 1995;376(6543):768-71.

3. Streit A, Stern CD, Thery C, Ireland GW, Aparicio S, Sharpe MJ, et al. A role for HGF/SF in neural induction and its expression in Hensen's node during gastrulation. Development. 1995;121(3):813-24

4. Andermarcher E, Surani MA, Gherardi E. Co-expression of the HGF/SF and c-met genes during early mouse embryogenesis precedes reciprocal 
expression in adjacent tissues during organogenesis. Dev Genet. 1996;18(3):254-66.

5. Boccaccio C, Comoglio PM. Invasive growth: a MET-driven genetic programme for cancer and stem cells. Nat Rev Cancer. 2006;6(8):637-45.

6. Di Renzo MF, Olivero M, Giacomini A, Porte H, Chastre E, Mirossay L, et al. Overexpression and amplification of the met/HGF receptor gene during the progression of colorectal cancer. Clin Cancer Res Off J Am Assoc Cancer Res. 1995; 1 (2):147-54.

7. Lee JH, Han SU, Cho H, Jennings B, Gerrard B, Dean M, et al. A novel germ line juxtamembrane Met mutation in human gastric cancer. Oncogene. 2000;19(43):4947-53.

8. Comoglio PM, Giordano S, Trusolino L. Drug development of MET inhibitors: targeting oncogene addiction and expedience. Nat Rev Drug Discov. 2008;7(6):504-16.

9. Peng Z, Zhu Y, Wang Q, Gao J, Li Y, Li Y, et al. Prognostic significance of MET amplification and expression in gastric cancer: a systematic review with meta-analysis. PLoS One. 2014;9(1):e84502.

10. Mesteri I, Schoppmann SF, Preusser M, Birner P. Overexpression of CMET is associated with signal transducer and activator of transcription 3 activation and diminished prognosis in oesophageal adenocarcinoma but not in squamous cell carcinoma. Eur J Cancer. 2014;50(7):1354-60.

11. Qin YR, Li YX, Wang LD, Fan ZM, Li JL, He X, et al. Expression of c-myc, hTERT and c-Met in esophageal squamous cell carcinoma and lymph node metastatic tissue. J Zhengzhou Univ (Med Sci). 2006;41(1):34-6.

12. Zhang $X$, Shen L, Li J, Li Y, Li J, Jin M. A phase II trial of paclitaxel and cisplatin in patients with advanced squamous-cell carcinoma of the esophagus. Am J Clin Oncol. 2008;31(1):29-33.

13. Spigel DR, Edelman MJ, Mok T, O'Byrne K, Paz-Ares L, Yu W, et al. Treatment rationale study design for the metlung trial: a randomized, double-blind phase iii study of onartuzumab (MetMAb) in combination with erlotinib versus erlotinib alone in patients who have received standard chemotherapy for stage IIIB or IV met-positive non-small-cell lung cancer. Clin Lung Cancer. 2012;13(6):500-4.

14. Anderson MR, Harrison R, Atherfold PA, Campbell MJ, Darnton SJ, Obszynska J, et al. Met receptor signaling: a key effector in esophageal adenocarcinoma. Clin Cancer Res Off J Am Assoc Cancer Res. 2006;12(20 Pt 1):5936-43.

15. Dong G, Lee TL, Yeh NT, Geoghegan J, Van Waes C, Chen Z. Metastatic squamous cell carcinoma cells that overexpress c-Met exhibit enhanced angiogenesis factor expression, scattering and metastasis in response to hepatocyte growth factor. Oncogene. 2004;23(37):6199-208.

16. Engelman JA, Zejnullahu K, Mitsudomi T, Song Y, Hyland C, Park JO, et al. MET amplification leads to gefitinib resistance in lung cancer by activating ERBB3 signaling. Science. 2007;316(5827):1039-43.

17. Bardelli A, Corso S, Bertotti A, Hobor S, Valtorta E, Siravegna G, et al. Amplification of the MET receptor drives resistance to anti-EGFR therapies in colorectal cancer. Cancer Discov. 2013;3(6):658-73.

18. Shattuck DL, Miller JK, Carraway 3rd KL, Sweeney C. Met receptor contributes to trastuzumab resistance of Her2-overexpressing breast cancer cells. Cancer Res. 2008;68(5):1471-7.

19. Minuti G, Cappuzzo F, Duchnowska R, Jassem J, Fabi A, O'Brien T, et al. Increased MET and HGF gene copy numbers are associated with trastuzumab failure in HER2-positive metastatic breast cancer. Br J Cancer. 2012;107(5):793-9.

20. Chen CT, Kim H, Liska D, Gao S, Christensen JG, Weiser MR. MET activation mediates resistance to lapatinib inhibition of HER2-amplified gastric cancer cells. Mol Cancer Ther. 2012;11(3):660-9.

\section{Submit your next manuscript to BioMed Central and take full advantage of:}

- Convenient online submission

- Thorough peer review

- No space constraints or color figure charges

- Immediate publication on acceptance

- Inclusion in PubMed, CAS, Scopus and Google Scholar

- Research which is freely available for redistribution 